\title{
Outpatient Commitment on the Ground: Listening to Consumers and Providers
}

\author{
Christopher Gjesfjeld \\ Michaela Kennedy
}

\begin{abstract}
Current debate and research on outpatient commitment (OPC) has examined whether OPC is (1) clinically effective or (2) ethically acceptable, yet little research has sought the voices of consumers and mental health providers who are most intimately impacted by outpatient commitment. Our research was specifically interested in the perspective that consumers and providers had about OPC. Qualitative interviews were conducted with nine consumers on OPC orders and eight treatment providers associated with these consumers. Three major themes emerged. First, consumers voiced an ambiguous sense of personal control in the context of OPC orders. Second, consumers and mental health providers maintained inconsistent understandings of outpatient commitment. Finally, all consumers reported an improvement in their life after being on OPC. Based on these findings, we suggest methods by which mental health providers could facilitate a collaborative relationship with consumers despite working within a context of OPC orders.
\end{abstract}

Keywords: Involuntary commitment, coercion, community mental health services, mandated treatment, OPC

\section{INTRODUCTION}

Outpatient commitment (OPC) has been defined as "a form of civil commitment in which the court orders an individual to comply with a specific outpatient treatment program” (Torrey \& Kaplan, 1995). With outpatient commitment laws in over 40 states in the United States of America, the intention of OPC is to mandate outpatient mental health services to individuals with a serious mental illness who maintain a capacity for violence, suicide, or re-hospitalization if left untreated. Advocates of OPC emphasize the importance of appropriate mental health treatment for the individual and the protection of the community from violence (Torrey \& Zdanowicz, 2001). They note legal pressure requiring treatment is necessary for some individuals with mental illness because of their inability to make appropriate decisions about their own mental health care (Munetz, Galon, \& Frese, 2003). Geller (1986; 2006), for example, has argued that some intrusion into personal liberties may actually increase personal freedom; those who are provided treatment early in their illness may avoid involuntary inpatient hospitalization.

Opponents, however, see the utilization of OPC as problematic for a number of reasons. Some opponents contend that OPC undermines the aims of collaboration between the worker and client because of the "monitoring" and heightened stigma associated with OPC orders (Allen \& Smith, 2001; Bazelon Center for Mental Health Law, 2001). Other opponents have argued that mandated treatment, by its very existence,

Christopher Gjesfjeld, Ph.D., LCSW, is an Assistant Professor in the Department of Social Work at the University of North Dakota in Grand Forks. Michaela Kennedy, MA, LPC, is a Research Specialist at Family Services of Western Pennsylvania.

Copyright (C) 2011 Advances in Social Work Vol. 12 No. 2 (Fall 2011), 152-163 
represents the failure of a public mental health system that is underfunded and offers ineffective treatments (United States Psychiatric Rehabilitation Association, 2007). Opponents to OPC have also pointed out that longitudinal data refutes the assertion that mental illness is an independent contributor to violence, consequently making the necessity of OPC less relevant (Elbogen \& Johnson, 2009).

Besides ethical concerns surrounding mandating mental health treatment, another strand of OPC research has examined the specific clinical outcomes associated with outpatient commitment. Commonly known as the Duke study, Swartz et al. (2001) followed 331 involuntary patients randomly assigned to either voluntary or involuntary services after leaving the hospital. When individuals in this study were on OPC for over six months and received frequent services (over 7 professional contacts a month), various researchers within this Duke group found the individuals were less likely to be homeless, less likely to be a strain on caregivers, and had a greater quality of life (Compton et al., 2003; Groff et al., 2004; Swanson, Swartz, Elbogen, Wagner, \& Burns, 2003). These gains were only made, however, if mandated treatment was provided over an extended period of time and consisted of frequent visits with mental health providers.

On the other hand, a Cochrane Review that included this Duke study as well as an evaluation of OPC conducted in New York City found little evidence for the effectiveness of OPC (Kisely, Campbell, \& Preston, 2011; Steadman et al., 2001). The authors found few differences on health service use, social functioning, mental state, quality of life, or satisfaction with care between consumers on or off outpatient commitment orders. In the most recent analysis of the New York City study, researchers found a few potential benefits in certain clinical outcomes yet note "caution against using our results to justify an expansion of coercion in psychiatric treatment" (Phelan, Sinkewicz, Castille, Huz, \& Link, 2010).

In sum, the ethical and clinical effectiveness debates will most likely not be resolved (O’Reilly, 2004). Due to this mixed picture, we (an academic-agency research collaboration) found it difficult to advise a community-based agency providing mental health services in Western Pennsylvania about "best practices" in terms of OPC. However, we found common agreement that the current discourse on OPC lacked the voices of those most intimately connected to OPC orders: consumers and those who provide treatment. With this in mind, we sought to hear how consumers and providers experienced outpatient commitment. By hearing from individuals and treatment providers about their experience with outpatient commitment orders, we hoped to achieve a better understanding of how OPC "looked on the ground". We hoped our efforts could facilitate two aims. First, the voices of consumers and providers could stimulate an inter-agency dialogue about the role of OPC in the agency. Second, our analysis could add a different dimension to the current discussions concerning the ethical dilemmas and clinical effectiveness of OPC orders. 


\section{METHODOLOGY}

\section{Conceptual Framework}

A qualitative research method was selected as the most effective way to more deeply explore the different perspectives of both consumers ${ }^{1}$ and providers involved in OPC. A semi-structured interview format was chosen because consumer and provider perspectives were to be used to directly inform decision-making by agency administration. Given this evaluative purpose, sensitizing concepts, or "categories that the analyst brings to the data” (Patton, 2002a, p. 456), were identified and used to structure the interview data and as an aid for data analysis. These concepts included: the stakeholders' understandings of the OPC process, attitudes and corresponding feelings about being placed on outpatient commitment, and any feedback that could improve the current implementation of OPC. Due to the utilization of this inductive approach and the partnering with an agency employee to do this research, our work was consistent with Patton's (2002b) stance of pragmatic utilitarianism. He notes that this stance is vital when specific evaluative questions do not require a comprehensive philosophical, ontological, or epistemological frame. While this methodological approach is flawed in terms of providing specific knowledge that could be generalized to other agencies providing treatment under OPC orders, our inquiry was consistent with an approach providing specific yet descriptive answers to outpatient commitment stakeholders to inform them of the experiences of consumers and their mental health care providers.

We also attended a number of outpatient commitment hearings. These specific hearings were not specific to a particular consumer in our study but did help us understand the legal context of mandated treatment. These experiences aided us in asking appropriate questions to both providers and consumers as to their understanding of these hearings and their significance.

\section{Study Participants}

Nine interviews were conducted with consumers either currently or recently on OPC orders. Individuals were eligible for the study if they received services at Family Services, were involved in OPC either currently or during the previous 2 years, and were 18 years and older. Consumers were approached by clinical staff of Family Services either in-person or via mail. Individuals were given a brochure to sign if interested and informed of their eligibility for the study. Researchers contacted individuals who expressed an interest in the study and arranged a time and date to meet. All consumers who showed initial interest in the study completed the interview. Interviews were taperecorded and conducted either in an agency office or in the consumer's home. Interview sessions averaged about an hour in length and all participants received a $\$ 25$ gift card to a local grocery store for participating in the interview. Of the nine consumers who participated in the interviews, five were on an OPC order at the time of the interview, and

\footnotetext{
${ }^{1}$ The term "consumers" will be used to describe individuals with a serious mental illness who have been placed on outpatient commitment orders. This specific term, in the context of mandated treatment, is problematic given that this term implies a consumption of mental health services that is voluntary. And yet, this was the language used within this agency to describe clients receiving mental health services, voluntary or involuntary.
} 
four had been on an OPC order in the past two years. The age range of the nine individuals receiving services was age 28 to 71 . A total of five females and four males were interviewed and all were Caucasian. Eight of the nine consumers had been diagnosed with schizophrenia or schizoaffective disorder, whereas one consumer had a diagnosis that included both depressive and anxiety symptomatology.

After these nine consumers agreed to participate in the study, they were asked to voluntarily nominate a provider within Family Services that was intimately connected to their mental health care. Eight providers were nominated with one consumer who chose not to nominate a provider. The strategy of nomination was used for two reasons. First, we wanted to find providers that had some experience working with an individual on OPC orders. Second, we thought that this process of consumers nominating a provider would enable us get multiple perspectives on a particular consumer's experience with OPC. Providers nominated by consumers were approached with the study, provided informed consent, and contacted to arrange a time for meeting. All nominated providers participated in the research. These interviews were also recorded using digital recorders and conducted in a semi-structured format with identical concepts as used with the consumers. The professional titles of these providers included psychiatric nurses, case managers, and psychosocial rehabilitation counselors.

\section{Data Analysis}

After completion of the 17 interviews, all digital audio files were transcribed into written transcripts. Initial categories were defined by the three predetermined concepts about (1) understandings of the OPC process, (2) attitudes and corresponding feelings about the consumer being placed on outpatient commitment, and (3) feedback that could improve the current implementation of OPC. With these a priori categories, both researchers separately coded the written text, making notes in the margins and finding commonalities among them. After we developed our codes separately, we met weekly for a month to clarify what themes we saw as present within the transcripts keeping in mind our specific aim of informing agency decision-making pertaining to OPC. These themes were developed with the specific purpose of informing OPC stakeholders about how OPC intersects with the experiences of both providers and consumers.

\section{RESULTS}

In many ways, the opinions and feelings about OPC orders differed between consumers and agency treatment providers. Consumers often felt like they were being pushed into something they did not see a need for, whereas providers thought that outpatient commitment was helpful for consumers who lacked insight into their mental illness. Yet, common themes were present in both interviews: (1) the ambiguous location of personal control for consumers in mandated treatment, (2) divergent definitions of outpatient commitment provided by consumers and providers, and (3) a perceived personal benefit of outpatient commitment voiced by both consumers and providers. 


\section{“What Ever Happens, Happens”}

One of the predominant themes that emerged was the ambiguous location of personal control in mandated treatment. While it must be acknowledged that the very nature of mandated treatment suggests an individual has a compromised ability to assert selfcontrol, consumers' responses suggested a fatalistic stance to OPC orders whereby any personal self-control or initiative was of little use.

Consumer \#1: I never say nothing. I am afraid of the judge. I just agree with him. What ever happens, happens.

Consumer \#4: I kept saying to my caseworker when am I going to get off of this court order? I can be on my own to take my medications and make my appointments. I was pretty persistent. It felt like I wanted to hurry up and be responsible for myself. I felt that I was being treated a little childishly.

Consumer \#8: I have been on it five or six years and nothing has changed. They have not changed anything yet. I have the idea that they are not going to change it. Maybe six or seven years from now I will still be on it. I got the idea that I would like to get off of it, I would be better if I could get off of it. I think things would be better for me.

Consumer \#9: They want to treat me like a baby. 'How do you keep your house clean? Do you know how to wash your hair? Do you know how to bathe? Do you know how to do this?' Why don't they just let me live my life? They tell me I am not well and I am not doing good.

One treatment provider spoke at length about the reason consumers were not more involved in decisions about their own care.

Provider \#9: Anytime they hear 'hearing' and 'court order' or '302' or 'diversion' they are automatically thinking this is not a good thing. Often times they will sit there silently and not say anything, unless they are more intelligent and have been in the system. But often times the people who don't understand just sit there quietly and do as they are told. Often times we are their voice. But the question is: 'what if we are not there?' Then they are just kind of rolling along with whereever the doctor is putting them and giving them and pushing them. Often times individuals in mental health won't ask. They just assume that the doctors know what is right. Providers know what is right and they go with that. The majority [of consumers] . . have problems communicating and they have problems with authority so they won't speak up.

This excerpt illustrates how mental health providers can unintentionally influence the personal control of consumers on OPC orders. While providers perceive themselves as advocates for their client, the quote also suggests that this advocacy is necessary given the consumer's illness or intellectual capacity. While this is certainly a possibility, after observing several OPC hearings, it also appears reasonable that the passive stance of individuals may have some origin in the substantial legal jargon and formality of the process rather than being merely a consequence of psychiatric symptoms. 


\section{Inconsistent Understandings of OPC}

An inconsistent understanding of outpatient commitment was also a common theme among both providers and consumers. Consumers and providers alike could not accurately define OPC, the specific mandate of OPC orders, or the consequences of consumer non-compliance. Both consumers and providers were unsure or confused about how consumers get removed from OPC. Providers' descriptions of OPC varied as the following comments demonstrate:

Provider \#2: It's voluntary. Attending services that we can provide.

Provider \#3: To the best of my knowledge the outpatient commitment is only committing a person on a written agreement that they will take their medications and that they will keep their psychiatric appointments. Anything else is a bonus.

Provider \#7: My understanding is OPC at this agency is just in regards to the doctor's visit. Sometimes for therapy like a therapist.

Individuals subject to OPC also had a wide range of understandings about the definition of OPC:

Consumer \#1: Outpatient commitment means you are a patient out in the public. You can see a doctor anytime, stuff like that. To keep my nose clean and stay out of trouble. Take the medicine.

Consumer \#5: That is just where the doctor goes in and makes sure that I am obeying what she prescribes in medicine and makes sure that I come back to see her again. I don't understand the whole procedure but I understand that it is the court that I go to.

Consumer \#9: I am being treated by the court. The court pays for the treatment or something? I am treated but I am an outpatient. I don't have to be committed all the time.

In addition to being unclear about what OPC actually entailed, it was unclear to most consumers as to why they were continued on OPC despite taking their medication and living independently in the community. They noted that decisions about OPC did not appear to examine their current ability to keep appointments, take medication, and maintain daily activities. They also seemed uninformed about what factors influenced the decision-making process for the professionals to get them dropped from an OPC.

Consumer \#4: I can't remember who decided or what it was based on to let me finally go home. It was all of a sudden. The judge decided . . . Oh I know she said that they had a separate meeting between the nurse, where I worked, the case worker and maybe the judge and they said I was doing well enough that I did not need this court order.

Consumer \#5: I don't understand it [OPC process] but eventually the doctor wanted me to be off if it. They were going to decide what happens to me. They just looked at me and made the decision. It might even be made before I get there [to court hearing]. Before the doctor even met me she had all the previous notes 
from the previous doctor or from somebody. They already had their minds made up. She told me on the very first meeting that she is sure that I should keep coming to see her.

Consumer \#6: Sometimes they just take you off of it, [that's how] you know if you are doing good. If they think everything is alright they will take you back off of it.

For the consumer on OPC, there was little understanding about what behaviors or actions could assist them in being removed from outpatient commitment. They were confused about how the decisions were made to terminate OPC. Termination from OPC was something that just happened and was suddenly determined by professionals. Similarly, the providers interviewed had divergent understandings about the definition of OPC, the services that it mandated, and the consequences for individuals who did not follow the court order.

\section{A Personal Benefit}

Another consistent theme was that all individuals subject to outpatient commitment interviewed reported some improvement in their quality of life while receiving treatment under outpatient commitment orders. However, it was not consistently understood from individuals what aspect of outpatient commitment actually helped them improve. While some individuals noted that the treatment received was beneficial, others noted that available support and the access to mental health services was what ultimately helped them succeed.

Consumer \#1: Keeps me out of trouble. Keeps me on my medicine. Keeps me on an even keel.

Consumer \#6: I have been on court commitment many times and they always seem to work when I am on them. When I am taken off I end up back in the hospital soon after. It helped, it kept me going to my doctor appointments and it kept me medicated long enough to realize that I did have a problem.

Consumer \#7: I should be on court commitment. Now I have a car, I have an apartment, I have furniture, I have a job and I am stable and I agree with the court commitment's decisions. So my life is good right now compared to what it has been.

Consumer \#8: It helped me. I kind of got on my feet a little bit. If I did not have the program or treatment I would be back in the hospital or gutter or up at Torrance [State Hospital]. I have not been in the hospital for 6 years now.

Some individuals pointed out the understanding and availability of staff as the most helpful to them.

Consumer \#3: She [therapist] was really like god sent to me. I don't know if I would have made it without her. She just talked to me about everything. She understood everything I would tell her about what my problems were. And she would find a resolution for me. 
Consumer \#4: I think the best part of the outpatient commitment was that I saw a caseworker once a month. I could call her if I needed to. I was taking medication and that helped and I felt more responsible for my mother and I was fully in charge of taking care of my mother.

Despite treatment being mandated, individuals subject to OPC reported that they were doing better now. While it was not clear how the court order helped, the consumers reporting maintaining positive relationships with their treatment providers had a particularly important positive impact on their lives.

\section{Limitations}

Despite being able to interview nine individuals who had been on outpatient commitment, a number of individuals were uninterested or unwilling to participate in this study. It is possible that these individuals may not have had positive experiences on outpatient commitment or feared that this study would impact their court order status in a negative way. Due to symptoms common to psychotic disorders, it is also possible that individuals were reluctant to participate for fear of being placed on outpatient commitment, being hospitalized, or being reconnected with the mental health system. The specific characteristics of those willing to share their experiences certainly may not be representative of those typically on OPC orders. Consumers who were uninterested in sharing their experiences may have had more intense psychiatric symptoms or very different experiences with outpatient commitment than the consumers participating in our research.

Likewise, as a group, consumers who shared their experiences about OPC may have been either exceedingly positive or negative about the provider they selected for us to talk with. Despite the agency having personnel with a specific full-time role dedicated to evaluation and research independent of the specific clinical care these consumers received, consumers and providers may have shielded us from specific negative comments about their treatment under a court order. Our study was also limited in terms of the racial and geographic diversity of the participants; all consumers and their providers were Caucasian and resided in Western Pennsylvania. Further research on how consumers and providers, particularly from underrepresented racial and ethnic minorities, interact with mandated treatment would improve the current body of research relevant to OPC orders.

\section{CONCLUDING REMARKS AND RECOMMENDATIONS}

Despite research that has considered the clinical effectiveness of OPC (Kisely et al., 2011), a much smaller amount of research has explored the consumer and provider perspectives associated with these court orders. With initial interest from a social service agency to understand how their specific consumers and treatment providers were influenced by outpatient commitment orders, we saw promise in listening to their perspectives and experiences. Three general areas of questions were the initial topics of inquiry: (1) understandings of OPC, (2) attitudes and feelings associated with being on 
OPC, and (3) feedback that could encourage agency improvement when commitment orders are used.

The first theme prevalent in the consumer responses was the lack of active engagement consumers had in the OPC process. Consumers voiced little control of the process and general confusion about what specifically was being asked of them while on these orders. While passivity may be a very natural response to a court order, we see this theme as particularly troubling because of the potential for this passive pattern to continue into their post-OPC care. Simply put, how will consumers be active collaborators in their treatment when they are familiar with treatment experiences in which they are typically passive and obedient?

To address consumer passivity, we see promise in practicing collaboration early in the commitment process. One specific opportunity can be in the formal OPC legal hearing. First, one-on-one time with legal representation could be arranged before the actual court hearing. If the individual is uncomfortable or intimidated by the hearing, a written statement could also be created prior to the hearing that could assist the individual in presenting his or her viewpoint without having to speak spontaneously. Agency providers can help consumers understand the court proceedings in simple language and encourage them to express their own perspective. One provider described soliciting consumers' voices:

Provider \#9 - I am always in contact with them and trying to explain to them and I am asking questions for them because there are a lot of things we don't understand . . . I am speaking to the staff. I am asking if there are alternatives. My goal is not to see them in a state hospital or to even court order them. I will speak to my client and say what would your ideal situation be? Lets come up with our plan and then we mediate between what our thoughts are and the social worker's at the hospital and what the team is. My goal is to explain what is going to happen to my client. Offer them a chance, plenty ahead of time saying if you have anything to say, you might want to jot down some thoughts. We will review it because you do have a right to speak. If they want to speak to the attorney, we will pull the attorney aside and say they would like to speak to you ahead of time and get them as much time as possible.

We were encouraged by this particular provider's engagement with consumers to understand and have a voice in the OPC hearing. Even so, our research concludes that consumers require more information about the details of their commitment orders as they receive pharmacological and psychological treatment. We see ongoing education about OPC as an opportunity to strengthen the therapeutic relationship prior to the termination of OPC.

In addition to the finding that consumers had little voice in the process, we also found that consumers generally did not understand what was being asked from them while on commitment orders. Both providers and consumers offered different requirements for being on OPC orders. Did these orders require them to meet with the psychiatrist, go to specific groups, or give them special access to providers? Consumers, in particular, were also confused about what was expected prior to termination of the OPC orders. 
Individuals were unsure of what behaviors would encourage the termination of their treatment orders. The orders seem to be terminated without a specific rationale.

We see these varied understandings as problematic because they can undermine the consumers from become partners in their care. As behavioral theorists have widely noted, a behavior is extinguished if it receives no reinforcement. If the removal of the commitment orders is not specifically associated with specific behaviors of the consumer, how are such orders helpful? While it could be argued that the vague goals of OPC could encourage greater clinical discretion about a specific consumer, a truly collaborative relationship, under commitment orders, would seem to require that therapeutic goals be specifically named and regularly evaluated. As noted by the divergent understandings of OPC by providers, the rationale for the use of OPC must also be communicated among treatment providers so there is consistency in its use within the agency and clarity for why it is being utilized.

Finally, whether individuals liked or disliked being coerced into treatment, all individuals reported an improvement of their life or a personal benefit from treatment. This positive reaction may have been the result of the accessibility and availability of support services, which are key components to the effectiveness of OPC (Appelbaum, 2005; Swartz \& Monahan, 2001). When OPC is utilized without appropriate funding for mental health services, it is likely an ineffective tool (Petrila \& Christy, 2008).

Our consumers' positive reaction is an important finding because many individuals subject to OPC will continue to have a mental health illness that requires extensive treatment services. Strong therapeutic relationships with providers, as well as services that are continuously financially funded, are vital to the future therapeutic partnerships between consumers and treatment providers. Providers should be aware that the partnership during OPC can impact the future engagement of consumers with mental health care, either positively or negatively.

\section{References}

Appelbaum, P. S. (2005). Assessing Kendra's Law: Five years of outpatient commitment in New York. Psychiatric Services, 56(7), 791-792.

Allen, M., \& Smith, V. F. (2001). Opening Pandora’s box: The practical and legal dangers of involuntary outpatient commitment. Psychiatric Services, 52(3), 342-346.

The Bazelon Center for Mental Health Law. (2001, July). Studies of outpatient commitment are misused. Washington, DC: Author. Retrieved from http://www.bazelon.org/Where-We-Stand/Self-Determination/ForcedTreatment/Outpatient-and-Civil-Commitment/Resources.aspx

Compton, S. N., Swanson, J. W., Wagner, H. R., Swartz, M. S., Burns, B. J., \& Elbogen, E. B. (2003). Involuntary outpatient commitment and homelessness in persons with severe mental illness. Mental Health Services Research, 5(1), 27-38.

Elbogen, E. B., \& Johnson, S. C. (2009). The intricate link between violence and mental disorder. Archives of General Psychiatry, 66(2), 152-161. 
Geller, J. L. (1986). Rights, wrongs, and the dilemma of coerced community treatment. American Journal of Psychiatry, 143(10), 1259-1264.

Geller, J. L. (2006). The evolution of outpatient commitment in the USA: from conundrum to quagmire. International Journal of Law and Psychiatry, 29(3), 234248.

Groff, A., Burns, B., Swanson, J., Swartz, M., Wagner, H. R., \& Thompson, M. (2004). Caregiving for persons with mental illness: The impact of outpatient commitment on caregiving strain. Journal of Nervous \& Mental Disease, 192(8), 554-565.

Kisely, S. R., Campbell, L. A., \& Preston, N. J. (2011). Compulsory community and involuntary outpatient treatment for people with severe mental disorders. Cochrane Database of Systematic Reviews, Issue 2. Art. No.: CD004408. DOI: 10.1002/14651858.CD004408.pub3.

Munetz, M. R., Galon, P. A., \& Frese, F. J., III. (2003). The ethics of mandatory community treatment. The Journal of the American Academy of Psychiatry and Law, 31(2), 173-183.

O’Reilly, R. (2004). Why are community treatment orders controversial? Canadian Journal of Psychiatry, 49(9), 579-584.

Patton, M. Q. (2002a). Qualitative research and evaluation methods. Thousand Oaks, CA: Sage.

Patton, M. Q. (2002b). Two decades of developments in qualitative inquiry: A personal, experiential perspective. Qualitative Social Work, 1(3), 261-283.

Petrila, J., \& Christy, A. (2008). Florida’s outpatient commitment law: A lesson in failed reform? Psychiatric Services, 59(1), 21-23.

Phelan, J. C., Sinkewcz, D. M., Castille, D. M., Huz, S., \& Link, B. G. (2010). Effectiveness and outcomes of assisted outpatient treatment in New York State. Psychiatric Services, 61(2), 137-143.

Steadman, H. J., Gounis, K., Denis, D., Hopper, K., Roche, B., Swartz, M., et al. (2001). Assessing the New York City outpatient commitment pilot program. Psychiatric Services, 52(3), 330-336.

Swanson, J. W., Swartz, M. S., Elbogen, E. B., Wagner, R., \& Burns, B. J. (2003). Effects of involuntary outpatient commitment on subjective quality of life in persons with severe mental illness. Behavioral Sciences and the Law, 21(4), 473-491.

Swartz, M. S., \& Monahan, J. (2001). Introduction: Special section on involuntary outpatient commitment. Psychiatric Services, 52(3), 323-324.

Swartz, M. S., Swanson, J. W., Hiday, V. A., Wagner, H. R., Burns, B. J., \& Borum, R. (2001). A randomized controlled trial of outpatient commitment in North Carolina. Psychiatric Services, 52(3), 325-329. 
Torrey, E. F., \& Kaplan, R. J. (1995). A national survey of the use of outpatient commitment. Psychiatric Services, 46(8), 778-784.

Torrey, E, F., \& Zdanowicz, M. (2001). Outpatient commitment: What, why, for whom? Psychiatric Services 52(3), 337-341.

United States Psychiatric Rehabilitation Association (2007, March). Position paper on involuntary outpatient commitment. Linthicum, MD. Retrieved from

http://knol.google.com/k/uspra-staff/position-paper-on-involuntary/9hcd4qaqyqq0/5\#

\section{Author note:}

Address correspondence to: Christopher Gjesfjeld, Ph.D., Gillette Hall Room 301C, 225

Centennial Drive Stop 7135, Grand Forks, ND 58202-7135. Email:

christopher.gjesfjeld@email.und.edu 